\title{
SYSTEMATIC NODAL DISSECTION IN THE INTRATHORACIC STAGING OF PATIENTS WITH NON-SMALL CELL LUNG CANCER
}

Alastair N. J. Graham, FRCS(Ed)

K. John M. Chan, MB

Ugo Pastorino, MD

Peter Goldstraw, FRCS
Objective: Although systematic nodal dissection is accepted as an important component of the intrathoracic staging of disease in patients undergoing thoracotomy for lung cancer, many surgeons still do not routinely perform it. We reviewed our practice to assess the information provided by its routine application even when lymph node metastases are considered unlikely. Methods: The records of 240 patients undergoing thoracotomy for clinically staged cT1-3 N0-1 non-small cell lung cancer were reviewed. In 5 cases $(2 \%)$ mediastinal dissection was not performed because of specific contraindications and in 8 cases (3\%) exploratory thoracotomy was performed. The pathologic findings in the 227 patients who underwent pulmonary resection with systematic nodal dissection were analyzed. Results: The median number of nodal stations, including N1 and N2, examined and submitted separately for histologic assessment was 7 per patient (range 3-13). N2 disease was disclosed in 46 patients overall ( $20 \%$ ), including 41 of the 227 patients undergoing pulmonary resection $(18 \%)$ and 5 of those undergoing exploratory thoracotomy $(62.5 \%)$. No subgroup had a $0 \%$ incidence of $\mathrm{N} 2$ metastases. Multivariate analysis showed that younger age, increasing tumor size, left lower lobe origin, and bronchial origin were significant independent variables for prediction of lymph node metastases at the N1 level, the N2 level, or both. Conclusions: Because no clinical or pathologic subset of patients with a negligible incidence of $\mathbf{N} 2$ disease could be discriminated, systematic nodal dissection must be routinely employed for accurate intrathoracic staging of non-small cell lung cancer. (J Thorac Cardiovasc Surg 1999;117:246-51)

\begin{abstract}
S ystematic nodal dissection is an essential component of the intrathoracic staging of disease in patients undergoing thoracotomy for lung cancer. It allows confirmation that a complete resection of all malignant tissue is possible and enables accurate determination of the extent of pulmonary resection required, thus minimizing sacrifice of functioning lung parenchyma. Because of the prognostic information it provides, it facilitates the surgeon's objective decision as to whether the risk of resection is balanced by the probability of cure, and it is
\end{abstract}

From the Department of Thoracic Surgery, Royal Brompton Hospital, London, United Kingdom.

Received for publication Jan 26, 1998; revisions requested April 15, 1998; revisions received Sept 3, 1998; accepted for publication Sept 16, 1998.

Address for reprints: P. Goldstraw, FRCS, Consultant Thoracic Surgeon, Department of Thoracic Surgery, Royal Brompton Hospital, Sydney St, London, SW3 6NP, United Kingdom.

Copyright () 1999 by Mosby, Inc.

$0022-5223 / 99 \$ 8.00+0 \quad \mathbf{1 2} / \mathbf{1} / \mathbf{9 4 6 4 0}$ essential in any trial of adjuvant or neoadjuvant therapy. However, a survey of surgeons performing lung cancer resections in the United Kingdom found that $45 \%$ of these surgeons would not routinely sample macroscopically normal mediastinal lymph nodes at thoracotomy, with only $23 \%$ routinely excising all mediastinal lymph nodes. ${ }^{1}$ Furthermore, enthusiasm for video-assisted techniques has led some surgeons to abandon systematic nodal dissection in their normal practice, with a recent suggestion that thoracoscopic resection without mediastinal node dissection might be adequate for some small, peripheral tumors. ${ }^{2}$

In the Royal Brompton Hospital we routinely excise and submit separately for histologic examination all accessible ipsilateral mediastinal nodes in all patients undergoing resection of lung cancer. If pneumonectomy is not required for resection of the primary tumor, the appropriate interlobar and lobar nodes are examined in a similar fashion to objectively determine the appropriate level of resection. We reviewed our findings with 
this policy to assess the information provided through its routine application, even when lymph node metastases are not suspected.

\section{Methods}

Patients. The cases of patients undergoing thoracotomy for resection of non-small cell lung cancer by a single consultant surgeon during the 5-year period starting in 1990 were found in the thoracic surgery database. During this time any patient who had been determined to have N2 disease before thoracotomy was not operated on, and neither neoadjuvant nor postoperative adjuvant therapy was used. After those undergoing resection of recurrent lung cancer or primary tracheal malignancies were excluded, 240 patients remained. Mediastinal dissection was contraindicated because of poor medical condition and limited resection options in 3 cases, because of previous mediastinal node dissection for a previous primary lung cancer in 1 case, and because of previous mediastinal irradiation in another case. In 8 patients $(3 \%)$ resection of the tumor was not undertaken after full assessment at thoracotomy, and 5 of these patients had N2 disease. In 3 of these patients systematic nodal dissection permitted the decision not to proceed with resection; 2 had multilevel N2 disease and 1 had limited $\mathrm{N} 2$ disease but with a risk of pneumonectomy that was considered to outweigh the chance of cure. The remaining patients underwent exploratory thoracotomy because of mediastinal invasion $(n=2)$, parietal pleural dissemination $(n=2)$, or previously undisclosed bilateral bronchoalveolar carcinoma in the presence of a resectable squamous carcinoma $(\mathrm{n}=1)$. There were thus 227 patients who underwent pulmonary resection with systematic nodal dissection, and analysis was restricted to this group because accurate assessment of tumor size and status of $\mathrm{N} 1$ nodes would be unavailable for those undergoing exploratory thoracotomy without resection. The mean age $( \pm \mathrm{SD})$ was $63.2 \pm 10.1$ years (range 35-87 years), and $175(77 \%)$ were male. Pneumonectomy was performed in $71(31 \%)$, lobectomy (including 14 bilobectomies) was performed in 138 (61\%), and segmentectomy was performed in $14(6 \%)$. A nonanatomic wedge resection was performed in 1 patient whose poor respiratory reserve precluded a more radical operation. Resections were extended to the chest wall or mediastinum in 13 patients $(6 \%)$, and bronchoplastic procedures were performed in 11 patients (5\%).

Clinical staging. All patients had disease clinically staged as cT1-3 N0-1. Initial assessment was conducted by history, examination, blood tests, and chest radiography. All patients underwent computed tomographic scan of the chest, abdomen, and brain. Isotope bone scans were performed in those with recent weight loss, anemia, bone pain, or elevated alkaline phosphatase or calcium levels. Cervical mediastinoscopy (supplemented by left anterior mediastinotomy if the tumor was in the left upper lobe) was selectively performed if the computed tomographic findings suggested invasion of the superior mediastinum or enlarged nodes $(>1.5 \mathrm{~cm}$ short axis) within reach of the mediastinoscope. Preoperative mediastinal exploration had been carried out in 75 (33\%) of the 227 patients who under- went pulmonary resection and systematic nodal dissection and in $5(62 \%)$ of the 8 patients who underwent exploratory thoracotomy. The numbers of lymph nodal stations sampled by mediastinoscopy ranged from 0 to 5 ; the median was 3 .

Intrathoracic staging. Our standard approach was to perform a posterolateral thoracotomy and assess the primary tumor. Histologic confirmation of the diagnosis, if not already available, was obtained by frozen-section analysis and the $\mathrm{T}$ stage was established. If the primary tumor was resectable (T1-3), the dissection of the mediastinal nodes was commenced before pulmonary resection. All nodes were stored on a piece of sterile card, labeled according to the Naruke map, ${ }^{3}$ and examined by the surgeon when the dissection was completed. Basic oncologic principles were adhered to, with the dissection continued along anatomic planes and crushing or transection of lymph nodes avoided. On the right side, stations $10,4,3$, and 2 were removed and then the hilum was dissected from posterior to inferior, removing stations 7, 8, and 9 . The dissection was continued anteriorly, mobilizing the superior pulmonary vein and main pulmonary artery. On the left side, the dissection was commenced at the aortic arch by locating the vagus nerve and its recurrent laryngeal branch and excising stations 5, 6, and 4; it then proceeded around the hilum as on the right side. When this was completed the surgeon was able to confirm that resection of the primary tumor by pneumonectomy was possible. Each lymph nodal station was serially sectioned in turn, with examination for the macroscopic appearance of metastases. Histologic confirmation of macroscopically involved nodes was obtained by frozen-section analysis if necessary. This allowed the surgeon to assess the possibility and advisability of complete resection of all intrathoracic tumor. If the primary tumor would permit a lesser resection by lobectomy or segmentectomy, the appropriate interlobar and lobar nodes were examined in a similar fashion to objectively determine the appropriate level of resection.

Statistical methods. Data on tumor size, N stage, histologic type and grade, and bronchial origin were obtained from the final pathology report. $\mathrm{T}$ stage and lobar origin were taken from the surgeon's operating notes and cross-referenced with the pathology report. Univariate analysis was performed, with the dependent variables being incidence of lymph node metastases ( $11+\mathrm{N} 2$ versus N0), incidence of mediastinal node metastases ( $\mathrm{N} 2$ versus $\mathrm{N} 1$ ), and presence of skip metastases in the mediastinal nodes (skip N2 versus metastases in both $\mathrm{N} 1$ and N2 nodes).

Contingency table data were examined for associations and row-column homogeneity by means of 2 -sided $\chi^{2}$ tests, with the trend test for proportions performed as described elsewhere. ${ }^{4}$ Continuous variables were assessed with the 2 -sided Student $t$ test. Multivariate analyses were based on logistic regression models fitted by stepwise backward progression $(P<.1)$ and also by stepwise forward progression $(P<.05)$. The dependent variable was incidence of lymph node metastases ( $\mathrm{N} 1$ and $\mathrm{N} 2$ versus $\mathrm{N} 0$ ). The independent variables were sex, age, whether a previous mediastinoscopy had been performed, the number of nodes sampled during any previous 
Table I. Patient and primary tumor details in 227 patients undergoing pulmonary resection and systematic nodal dissection

\begin{tabular}{|c|c|c|c|c|c|c|c|}
\hline & \multicolumn{2}{|c|}{$p N O(n=136)$} & \multicolumn{2}{|c|}{$p N 1(n=50)$} & \multicolumn{2}{|c|}{$p N 2(n=41)$} & \multirow[b]{2}{*}{ Total $(n=227)$} \\
\hline & Value & $S D$ or \%* & Value & SD or \%* & Value & $S D$ or $\% *$ & \\
\hline Mean age (y) & 64.8 & 9.9 & 61.4 & 10 & 60.5 & 10 & 63.3 \\
\hline \multicolumn{8}{|l|}{ Sex } \\
\hline Male & 102 & $58 \%$ & 40 & $23 \%$ & 33 & $19 \%$ & 175 \\
\hline Female & 34 & $65 \%$ & 10 & $19 \%$ & 8 & $15 \%$ & 52 \\
\hline \multicolumn{8}{|l|}{ Previous mediastinoscopy? } \\
\hline No & 100 & $66 \%$ & 31 & $21 \%$ & 20 & $13 \%$ & 151 \\
\hline Yes & 36 & $47 \%$ & 19 & $25 \%$ & 21 & $28 \%$ & 76 \\
\hline Mean tumor diameter $(\mathrm{cm})$ & 36.2 & 19.4 & 43.9 & 25 & 47.1 & 28.4 & 39.9 \\
\hline \multicolumn{8}{|l|}{ Cellular classification } \\
\hline Squamous cell & 62 & $57 \%$ & 25 & $23 \%$ & 22 & $20 \%$ & 109 \\
\hline Adenocarcinoma & 58 & $67 \%$ & 17 & $20 \%$ & 12 & $14 \%$ & 87 \\
\hline Large cell & 15 & $56 \%$ & 6 & $22 \%$ & 6 & $22 \%$ & 27 \\
\hline Adenosquamous & 1 & $25 \%$ & 2 & $50 \%$ & 1 & $25 \%$ & 4 \\
\hline \multicolumn{8}{|l|}{ T stage } \\
\hline $\mathrm{T} 1$ & 61 & $71 \%$ & 16 & $19 \%$ & 9 & $10 \%$ & 86 \\
\hline $\mathrm{T} 2$ & 66 & $54 \%$ & 27 & $22 \%$ & 29 & $24 \%$ & 122 \\
\hline T3 & 8 & $47 \%$ & 7 & $41 \%$ & 2 & $12 \%$ & 17 \\
\hline $\mathrm{T} 4$ & 1 & $50 \%$ & 0 & $0 \%$ & 1 & $50 \%$ & 2 \\
\hline \multicolumn{8}{|l|}{ Lobar origin } \\
\hline Right upper & 44 & $63 \%$ & 15 & $21 \%$ & 11 & $16 \%$ & 70 \\
\hline Right middle & 10 & $77 \%$ & 1 & $8 \%$ & 2 & $15 \%$ & 13 \\
\hline Right lower & 25 & $66 \%$ & 8 & $21 \%$ & 8 & $13 \%$ & 38 \\
\hline Left upper & 51 & $58 \%$ & 19 & $22 \%$ & 18 & $20 \%$ & 88 \\
\hline Left lower & 6 & $33 \%$ & 7 & $39 \%$ & 5 & $28 \%$ & 18 \\
\hline \multicolumn{8}{|l|}{ Location of primary $\dagger$} \\
\hline Peripheral & 70 & $73 \%$ & 14 & $15 \%$ & 12 & $12 \%$ & 96 \\
\hline Central & 62 & $49 \%$ & 36 & $28 \%$ & 29 & $23 \%$ & 127 \\
\hline
\end{tabular}

*SD for mean values, percentage of total (final column) for numbers of patients.

$\dagger$ In 4 patients the location of the primary could not be classified.

mediastinoscopy, the number of nodes sampled during the thoracotomy, tumor size, histologic type, histologic grade, $\mathrm{T}$ stage, lobe of origin, and whether the tumor was peripheral or central. Analysis was performed with SPSS Version 6.1 (SPSS Inc, Chicago, Ill).

\section{Results}

The median number of nodal stations, including N1 and N2, examined and submitted separately for histologic assessment was 7 per patient (range 3-13). The mean $( \pm$ SD) number of N1 nodal stations submitted separately increased from $1.4 \pm 0.9$ in the first year of the study to $2.7 \pm 1.3$ in the last $(P<.0001)$. The mean number of $\mathrm{N} 2$ nodal stations excised separately was 4.9 \pm 1.2 , and there was no significant change in this during the study period. Histologic confirmation of the surgeon's findings by frozen-section analysis was obtained for $\mathrm{N} 1$ nodes in 42 cases (19\%), N2 nodes in 54 cases (24\%), and the primary tumor in 125 cases $(55 \%)$.

Lymph node metastases. Table I shows the patient and primary tumor details for each surgical-pathologic lymph node stage. Univariate analysis showed that the following factors were significantly associated with node-positive tumors (N1 and N2): younger age $(P=$ $.005)$, previous mediastinoscopy $(P=.004)$, larger tumor diameter $(P=.005)$, increasing $\mathrm{T}$ stage $(P=.01)$, left lower lobe origin $(P=.03)$, and central location of tumor $(P=.0005)$. There were no significant differences in these factors between patients who had mediastinal node metastases and those who had N1 metastases. There was a significant trend toward increasing incidence of nodal metastases (N1 and N2) as the T stage increased from T1 to T3 $(P=.01)$ and as the tumor became more central, with the incidences associated with peripheral tumors and those arising from segmental, lobar, and main bronchi being $27 \%, 42 \%, 68 \%$, and $73 \%$, respectively ( $P$ $<.0001)$. There was no significant association between histologic type or grade of primary tumor and presence of lymph node metastases. Multivariate analysis showed that younger age, increasing tumor size, left lower lobe origin, and bronchial origin (no bronchial attachment versus segmental versus lobar versus main) were signif- 
Table II. Results of multivariate analysis: Significant findings

\begin{tabular}{lccc}
\hline Variable & $\beta$ & $S E$ & $\mathrm{P}$ \\
\hline Age (y) & -.037 & 0.015 & .01 \\
Tumor diameter (mm) & .016 & 0.006 & .01 \\
Lobe of origin & & & .12 \\
$\quad$ Upper versus middle & .331 & 0.247 & .18 \\
$\quad$ Middle versus lower & -.829 & 0.404 & .04 \\
Bronchial origin & .627 & 0.176 & $<.001$ \\
Constant & -.135 & 1.016 & .9 \\
\hline
\end{tabular}

Significant findings on multivariate analysis (forward and backward stepwise analysis gave identical results). $\beta$, Logistic regression coefficient.

icant independent variables for prediction of lymph node metastases (Table II). Interaction terms between these variables were all significant, except that between age and bronchial origin, confirming that the relationships among these variables and the incidence of lymph node metastases are complex.

Small, peripheral tumors. Because it has been suggested that thoracoscopic wedge resection without mediastinal node dissection may be adequate for some small, peripheral tumors, ${ }^{2}$ we examined the findings in this subgroup of patients (Table III). Sixty percent of peripheral tumors $2 \mathrm{~cm}$ or smaller in diameter were adenocarcinomas, compared with $35 \%$ of more central or larger tumors $(P=.03)$. Twenty-four percent of peripheral tumors $2 \mathrm{~cm}$ or smaller in diameter had lymph node metastases, with the incidence being $26 \%$ if $3 \mathrm{~cm}$ was used as the cutoff point.

Skip metastases. In 14 patients metastases were detected in mediastinal nodes with documented absence in N1 nodes. Table IV shows comparisons between the primary tumors in patients with these "skip metastases" and those with metastases in both N1 and N2 nodes. Skip metastases were more common with peripheral $(P=.04)$ and right-sided tumors $(P=.01)$. The sites of mediastinal lymph node metastases in patients with skip metastases and those with metastases in both $\mathrm{N} 1$ and N2 nodes are shown in Table V.

\section{Discussion}

Mediastinal node dissection has become accepted as an integral component of the intrathoracic staging of disease in patients undergoing resection of non-small cell lung cancer ${ }^{5}$ because the presence of metastases in mediastinal lymph nodes has been shown to be the most important indicator of a low chance of survival after resection, ${ }^{6,7}$ and all currently available modalities for assessing mediastinal lymph node status before the operation have inadequate sensitivities for their detection. 8,9 The accuracy of this clinical evaluation of the
Table III. Lymph node status in patients with small, peripherally based tumors*

\begin{tabular}{lrcc}
\hline & $p N O$ & $p N 1$ & $p N 2$ \\
\hline$\leq 2 \mathrm{~cm}$ & & & \\
Squamous cell & 8 & 0 & $1 \dagger$ \\
Adenocarcinoma & 11 & 3 & $1 \dagger$ \\
Large cell & 0 & 0 & 0 \\
Adenosquamous & 0 & 1 & 0 \\
$\leq 3 \mathrm{~cm}$ & & & \\
Squamous cell & 12 & 0 & $1 \dagger$ \\
Adenocarcinoma & 11 & 3 & $1 \dagger$ \\
Large cell & 3 & 1 & 2 \\
Adenosquamous & 0 & 1 & 0 \\
\hline
\end{tabular}

*Small, peripheral tumors (excluding those with a bronchial connection); that is, those that would be technically resectable by thoracoscopic wedge. $\dagger$ Skip metastasis.

lymph nodes by the surgeon at the time of the operation, supplemented by histologic confirmation by frozen-section analysis where appropriate, has already been demonstrated. ${ }^{9,10}$ Much confusion has been generated by the different terms, such as radical lymphadenectomy or lymph node sampling, used to describe lymph node dissection at thoracotomy. At an international workshop held at the National Heart and Lung Institute in London in October 1996 and supported by the International Association for the Study of Lung Cancer, a recommendation was made that the term systematic nodal dissection be used to describe the procedure that is most commonly carried out, a staging procedure consisting of dissection and examination of mediastinal, hilar, and lobar lymph nodes in a systematic fashion. ${ }^{11}$

In this series only $2 \%$ of patients had systematic nodal dissection omitted, in all cases because of specific contraindications. In only $3 \%$ was exploratory thoracotomy only performed, and this both compares well with the 10 to $15 \%^{12,13}$ rate in recent reports and reflects the thorough approach to preoperative staging in our unit, as previously documented. ${ }^{14}$ Because neoadjuvant chemotherapy was not routinely used during the period of this study, patients with $\mathrm{cN} 2$ disease confirmed at mediastinoscopy were treated with nonoperative therapy. At thoracotomy, N2 disease was disclosed in 46 patients overall (20\%), including 41 of the 227 patients undergoing pulmonary resection (18\%) and 5 of those undergoing exploratory thoracotomy $(62.5 \%)$. The incidence of $\mathrm{pN} 2$ disease was higher among those who had undergone previous mediastinoscopy, reflecting both the policy of its selective use and its lack of $100 \%$ sensitivity for discriminating patients with N2 disease.

There was a significant trend toward increasing incidence of lymph node metastases (N1 and N2) among 
Table IV. Skip N2 cases versus N2 cases without skip

\begin{tabular}{|c|c|c|c|c|}
\hline & \multicolumn{2}{|c|}{ Skip $(n=14)$} & \multicolumn{2}{|c|}{ No skip* $(n=27)$} \\
\hline & No. & $\%+$ & No. & $\%+$ \\
\hline \multicolumn{5}{|l|}{ Site of primary } \\
\hline Peripheral & 7 & 58 & 5 & 42 \\
\hline Central & 7 & 24 & 22 & 76 \\
\hline \multicolumn{5}{|c|}{ Cellular classification } \\
\hline Adenocarcinoma & 5 & 42 & 7 & 58 \\
\hline Squamous cell & 6 & 27 & 16 & 73 \\
\hline Large cell & 3 & & 3 & \\
\hline Adenosquamous & & & 1 & \\
\hline \multicolumn{5}{|l|}{ Side of primary } \\
\hline Right & 10 & 56 & 8 & 44 \\
\hline Left & 4 & 17 & 19 & 83 \\
\hline \multicolumn{5}{|l|}{ Lobar origin } \\
\hline Upper & 11 & 37 & 19 & 63 \\
\hline Middle & 0 & & 2 & 7 \\
\hline Lower & 3 & 33 & 6 & 67 \\
\hline
\end{tabular}

*Metastases in both $\mathrm{N} 1$ and $\mathrm{N} 2$ nodes.

$\dagger$ Percentage of row totals (skip + no skip).

patients with primary tumors that were more central, and multivariate analysis showed that younger age, increasing tumor size, left lower lobe origin, and bronchial origin were significant independent variables predicting lymph node metastasis. The effect of age probably represents a bias in patient selection, because elderly patients were less likely than young ones to be offered surgical treatment if the initial investigations showed that the tumor was probably of advanced stage. Interaction terms between these variables were all significant, except that between age and bronchial origin, confirming that there is a complex relationship among these variables. There were no significant predictors of stage $\mathrm{N} 2$ versus stage $\mathrm{N} 1$.

There has been recent debate regarding the need for mediastinal node dissection in patients with small, peripheral tumors, with a recent report from the National Cancer Centre in Tokyo documenting mediastinal lymph node metastases associated with $6.2 \%$ of peripheral, squamous tumors $2 \mathrm{~cm}$ in diameter or smaller and with $2.3 \%$ of those $3 \mathrm{~cm}$ in diameter or smaller with hilar nodes free of tumor. ${ }^{2}$ The authors suggested that mediastinal lymph node dissection might be omitted in these patients. We found these 2 subsets to have $\mathrm{pN} 2$ disease in $11 \%$ and $8 \%$ of cases, respectively, and we therefore believe that mediastinal dissection cannot be omitted in the case of small, peripheral tumors. An additional argument against this selective policy is that it depends on accurate cellular classification of the primary tumor, which is unlikely to be known before the operation for these small, bronchoscopically negative tumors and is not reliably deter-
Table V. Sites of mediastinal lymph node metastases (N2 stations)

\begin{tabular}{lcccc}
\hline Site of primary & $\begin{array}{c}\text { Upper } \\
(2-4)\end{array}$ & $\begin{array}{c}\text { Aortic } \\
(5-6)\end{array}$ & $\begin{array}{c}\text { Inferior } \\
(7-9)\end{array}$ & Total \\
\hline N2, no skip* & & & & \\
RUL & 5 & & 1 & 5 \\
RML & 1 & & 2 & 2 \\
RLL & & & 1 & 1 \\
LUL & 8 & 10 & 4 & 14 \\
LLL & 2 & 3 & 5 & 5 \\
Skip N2 & & & & \\
RUL & 6 & & 3 & 3 \\
RLL & 1 & & & 4 \\
LUL & 2 & 3 & &
\end{tabular}

$R U L$, Right upper lobe; $R M L$, right middle lobe; $R L L$, right lower lobe; $L U L$, left upper lobe; $L L L$, left lower lobe.

* Metastases in both N1 and N2 nodes.

mined by frozen-section analysis. For all patients with peripheral tumors $2 \mathrm{~cm}$ in diameter or smaller, the incidence of $\mathrm{N} 2$ disease was $8 \%$, similar to the range of $8 \%$ to $12 \%$ previously reported. ${ }^{2,15-17}$

Skip metastases occurred in $34 \%$ of patients with N2 disease, similar to the recently reported range of $29 \%$ to $33 \% .{ }^{18-20}$ Right-sided primary tumors were significantly more likely to be associated with skip metastases $(P=.01)$, and this has previously been seen in some studies ${ }^{20}$ but not in others. ${ }^{18,19}$ The reasons for this association are unclear but do not appear to include sampling error, because the mean number of N1 levels sampled was greater on the right side (data not shown). Although the incidences of skip metastases were similar for peripheral and central tumors at $7 \%$ and $5 \%$, respectively, there was an increased incidence of $\mathrm{N} 2$ metastases associated with $\mathrm{N} 1$ metastases for central tumors (17\%) compared with peripheral tumors $(5 \%, P$ $=.01)$. Few other studies have addressed this issue, but those that have done so have had similar findings. ${ }^{19}$ This decreased propensity of central tumors toward skip metastases may be explained by the findings of direct lymphatic pathways to mediastinal nodes by injection of the peripheral subpleural lymphatics in cadavers. ${ }^{21}$ The significant incidence of skip metastases from peripheral tumors reinforces the need for a full lymph node dissection in these patients.

The sites of mediastinal metastases found essentially confirm the work of previous investigators in this field. ${ }^{2,3,16}$ Metastases to subcarinal nodes occurred with primary tumors in all lobes, which would support the contention that these nodes should always be excised regardless of the site of the primary tumor. ${ }^{22}$

There has been some concern regarding possible adverse effects of mediastinal node dissection, with a 
suggestion that it may predispose the patient toward the development of acute respiratory distress syndrome. The overall incidences according to strict diagnostic criteria ${ }^{23}$ of acute respiratory distress syndrome and acute lung injury among the patients undergoing pneumonectomy and lobectomy were $8 \%$ and $3 \%$, respectively. Precise figures for the development of these conditions after lung resection without mediastinal dissection are not available for comparison, although the higher incidence after pneumonectomy than lobectomy with an identical mediastinal dissection seen in this and other studies ${ }^{24}$ suggests that the volume of lung resected, rather than the mediastinal dissection, is the main risk factor. Another concern raised has been a possible adverse effect on the immunologic response to dissemination of cancer cells, with a report of lower survival among patients with T1 N0 M0 tumors who underwent complete ipsilateral mediastinal node dissection than among those who underwent hilar node dissection and mediastinal node sampling. ${ }^{25}$ Because patients in that study were not randomly assigned to treatment groups, however, there was a potential for bias in the selection of patients for mediastinal dissection. In addition, the number of nodal stations "sampled" was not reported. The true potential of mediastinal node dissection to predispose the patient toward these 2 possible adverse effects can only ever be determined by a randomized, controlled trial.

We found that the routine application of systematic nodal dissection to patients with disease clinically staged as cN0-1 found significant incidence of pN2 in every subset analyzed. We therefore believe that systematic nodal dissection must be routinely employed at thoracotomy if the intrathoracic stage of disease in patients with non-small cell lung cancer is to be accurately determined.

We thank Dr Gordon W. Cran, $\mathrm{PhD}$, for statistical advice.

\section{REFERENCES}

1. Tsang GM, Watson DC. The practice of cardiothoracic surgeons in the perioperative staging of non-small cell lung cancer. Thorax 1992;47:3-5.

2. Asamura H, Nakayama H, Kondo H, Tsuchiya R, Shimosato Y, Naruke T. Lymph node involvement, recurrence, and prognosis in resected small, peripheral, non-small-cell lung carcinomas: are these carcinomas candidates for video-assisted lobectomy? J Thorac Cardiovasc Surg 1996;111:1125-34.

3. Naruke TJ, Suemasu K, Ishikawa S. Lymph node mapping and curability at various levels of resected lung cancer. J Thorac Cardiovasc Surg 1978;76:832-9.

4. Altman DG. Practical statistics for medical research. London: Chapman and Hall; 1997. p. 263.

5. Goldstraw P, Rocmans P, Bali D, et al. Pretreatment minimal staging for non-small cell lung cancer: an updated consensus. Lung Cancer 1994;11:S1-4.
6. Mountain CF. Value of the new TNM staging system for lung cancer. Chest 1989;96:47S-9S.

7. Naruke T, Goya T, Tsuchiya R, Suemasu K. Prognosis and survival in resected lung cancer based on the new international staging system. J Thorac Cardiovasc Surg 1988;96:440-7.

8. Webb RW, Gatsonis C, Zerhouni EA, et al. CT and MR in staging non-small cell bronchogenic cancer: report of the radiological diagnostic oncology group. Radiology 1991;178:70513.

9. Izbicki JR, Thetter O, Karg O, et al. Accuracy of computed tomographic scan and surgical assessment for staging of bronchial carcinoma. J Thorac Cardiovasc Surg 1992;104:413-20.

10. Gaer JA, Goldstraw P. Intraoperative assessment of nodal staging at thoracotomy for carcinoma of the bronchus. Eur J Cardiothoracic Surg 1990;4:207-10.

11. Goldstraw P. Report on the International Workshop on Intrathoracic Staging. Lung Cancer 1997;18:107-11.

12. Steinbaum SS, Uretzky ID, McAdams HP, Torrington KG, Cohen AJ. Exploratory thoracotomy for nonresectable lung cancer. Chest 1995;107:1058-61.

13. White PG, Adams H, Crane MD, Butchart EG. Preoperative staging of carcinoma of the bronchus: can computed tomographic scanning reliably identify stage III tumours? Thorax 1994;49: 951-7.

14. Fernando HC, Goldstraw P. The accuracy of clinical evaluative intrathoracic staging in lung cancer as assessed by postsurgical pathologic staging. Cancer 1990;65:2503-6.

15. Sagawa M, Saito Y, Takahashi S, et al. Clinical and prognostic assessment of patients with resected small peripheral lung cancer lesions. Cancer 1990;66:2653-7.

16. Ishida T, Yano T, Maeda K, Kaneko S, Tateishi M, Sugimachi K. Strategy for lymphadenectomy in lung cancer three centimeters or less in diameter. Ann Thorac Surg 1990;50:708-13.

17. Nonomura A, Mizukami Y, Shimizu J. Clinicopathological study of primary malignant tumours of the lung. J Surg Oncol 1995; 58:5-11.

18. Yoshino I, Yokoyama H, Yano T. Skip metastasis to the mediastinal lymph nodes in non-small cell lung cancer. Ann Thorac Surg 1996;62:1021-5

19. Libshitz HI, McKenna RJ, Mountain CF. Patterns of mediastinal metastases in bronchogenic carcinoma. Chest 1986;90:229-32.

20. Tateishi M, Fukuyama Y, Hamatake M. Skip mediastinal lymph node metastasis in non-small cell lung cancer. J Surg Oncol 1994; 57:139-42.

21. Riquet M, Hidden G, Debesse B. Direct lymphatic drainage of lung segments to the mediastinal nodes. J Thorac Cardiovasc Surg 1989;97:623-32.

22. Anonymous. Should subcarinal lymph nodes be routinely examined in patients with non-small cell lung cancer? The Lung Cancer Study Group. J Thorac Cardiovasc Surg 1988;95:883-7.

23. Bernard GR, Artigas A, Brigham KL, et al. The AmericanEuropean Consensus Conference on ARDS. Am J Respir Crit Care Med 1994;149:818-24.

24. Waller DA, Gebitekin C, Saunders NR, Walker DR. Noncardiogenic pulmonary edema complicating lung resection. Ann Thorac Surg 1993;55:140-3.

25. Funatsu T, Matsubara Y, Ikeda, S et al. Preoperative mediastinoscopic assessment of $\mathrm{N}$ factors and the need for mediastinal lymph node dissection in T1 lung cancer. J Thorac Cardiovasc Surg 1994;108:321-8. 